\title{
Monitoring Severe Head Injury: a Comparison of EEG and Somatosensory Evoked Potentials
}

\author{
Richard J. Moulton, Jennifer I.M. Brown and Stefan J. Konasiewicz
}

\begin{abstract}
We report on our experience with long-term monitoring of the EEG power spectrum and somatosensory evoked potentials (SSEPs) in 103 patients with severe closed head injury (Glasgow Coma Scale - GCS $\leq 8$ ). Patients were monitored for an average of 5 days post injury and monitoring was terminated when they died, regained consciousness or their intracranial physiologic parameters (primarily intracranial pressure - ICP) were stable for 2-3 days. Patients were treated according to a standard protocol that included mechanical ventilation, sedation, and neuromuscular blockade. At 7 of 9 twelve hour time intervals post injury, SSEPs were significantly $(p<.05)$ different between outcome groups using the Glasgow Outcome Score collapsed to 3 categories. The percent slow (delta) activity in the EEG was not significantly different between outcome groups at any time point, post injury. The total power in the EEG power spectrum differed only at the last time epoch post injury (108 hr.). Based on the superior prognostic capabilities of the SSEP, we routinely base critical management decisions on SSEP values. We have not been able to rely on EEG parameters for these same decisions due to the lack of clear distinction between good and poor prognosis groups based on common EEG parameters.
\end{abstract}

RÉSUMÉ: Surveillance des traumatismes crâniens sévères: comparaison de l'EEG et des potentiels évoqués somesthésiques. Nous rapportons notre expérience de surveillance à long terme du spectre de puissance EEG et des potentiels évoqués somethésiques (PESs) chez 103 patients ayant subi un traumatisme crânien fermé (échelle de coma de Glasgow $\leq 8$ ). Nous avons surveillé les patients pendant en moyenne 5 jours après la lésion et la surveillance était interrompue au décès, si le patient reprenait conscience ou si leurs paramètres physiologiques intracrânients (principalement la pression intracrânienne - PIC) étaient stables dupuis 2 ou 3 jours. Les patients étaient traités selon un protocole standard qui incluait la ventilation mécanique, , la sédation et le blocage neuromusculaire. Au septième du neuf intervalles de 12 heures après la lésion, les PESs étaient significativement différents $(p<0.05)$ entre les groupes selon leur évolution évaluée au moyen de l'échelle de Glasgow réduite à trois catégories. Le pourcentage d'activité lente (delta) n'était pas significativement différent entre les groupes selon leur évolution, quelque soit le dernier intervalle de temps après le lésion (108 hrs). Comme la valeur pronostique des PESs est supérieure, nos décisions critiques de traitement sont habituellement basées sur les valeurs de PESs. Nous n'avons pas pu nous fier sur les paramètres EEG pour prendre ces décisions à cause de l'absence de distinction claire entre le groupe avec un bon pronostic et celui avec un mauvais pronostic sur la base de paranlètres EEG communs.

Can. J. Neurol. Sci. 1998; 25: S7-S11

Given the limitations of the clinical neurological examination in comatose head injury patients, an objective, reliable measure of neurologic function in severely head-injured patients is desirable to monitor patient progress or lack thereof, the impact of secondary insults on neurologic function, and to measure the immediate effect of therapeutic interventions. In this paper we report on our experience with long-term monitoring (average 5 days) of the EEG power spectrum and somatosensory evoked potentials in a group of severely head injured patients and discuss the impact of monitoring these electrophysiologic parameters on the management of this patient group. EEG and somatosensory evoked potentials are compared by their respective abilities to predict short- and long-term patient outcomes.
Given the absence of a contemporaneous "gold standard" measure of cerebral function against which EEG and SSEPs can be compared, patient outcome is employed as a surrogate. Implicit is the assumption that patient outcome depends on some aspect(s) of cerebral function that can be measured early after injury and the extent to which a technique can predict outcome is a reflection of the fidelity with which that particular technique can measure critical aspects of cerebral physiology.

\footnotetext{
From the Division of Neurosurgery, St. Michael's Hospital and the University of Toronto. Toronto, Ontario.

Reprint requests to: $\mathrm{R}$. Moulton, Division of Neurosurgery, 38 Shuter Street, Toronto,
} Ontario. Canada M5B IA6 
The electroencephalogram has been employed in the investigation of traumatic coma virtually since its inception. Coma is generally associated with slowing of the background frequencies with the amount of slowing generally proportionate to the depth of coma. ${ }^{1}$ The major technical difficulties associated with use of conventional EEG as a monitoring tool for comatose patients involve the tremendous amount of data generated and the requirement for expert interpretation of the data at the bedside. A number of authors have attempted to deal with the problems of data reduction and simplification of EEG output.

The most recent and widely accepted form of data compression is power spectrum analysis of the EEG. The latter is a mathematical transformation of the EEG (or other complex wave phenomena) from the time to the frequency domain. The development, miniaturization and constant improvement of computer hardware have permitted the widespread availability at reasonable cost of computerized EEG monitoring equipment for what was formerly a very computationally demanding technique. Various graphic displays have evolved to present the frequency information, usually on a video terminal. The most frequently used measure has been the compressed spectral array pioneered by Bickford. ${ }^{2}$

Other authors have shown a relationship between compressed spectral patterns and outcome from coma. As with conventional EEG the amount of slowing in the background of the EEG is related to the depth of coma. Outcome can be predicted to a fair degree of accuracy from patterns observable on the compressed spectral array. In general, slow monotonous records are associated with a worse prognosis than fast, changeable records. ${ }^{3}$ In these studies the degree of prognostic accuracy spectral analysis of the EEG does not exceed that achievable from the clinical parameters of patients' age and GCS at admission to hospital. ${ }^{4,5}$

Investigators have also established a relationship between evoked potentials and outcome from head injury. Perhaps more importantly, evoked potentials have been shown to change in clinically meaningful ways when monitored over time.${ }^{6,7}$ Of the commonly measured evoked potentials, somatosensory (SSEPs), have been shown to bear the greatest relationship to patient outcome. $^{8}$ For this reason we have concentrated our efforts on the collection and analysis of SSEPS.

The most significant feature(s) of the SSEP is subject to debate. Outcome has been well correlated with central conduction time, number of peaks, longest latency activity in the SSEP, total amplitude in the SSEP, and more complex subjective grading systems. Certainly features of the SSEP that are dependent upon early latency components are the most reproducible both within and among individuals. The central conduction time (cervico-medullary junction to primary somatosensory cortex) is one such measure that has been extensively employed and clearly distinguishes between survivors and non-survivors from head injury. Loss of all cortical activity bilaterally in the SSEP is uniformly associated with death or survival in a severely debilitated state. However, it is the intermediate and long latency activity (70 msec. and beyond) that best distinguishes functional status among survivors. This appears to be true both in head injury patients 7.8 and in patients suffering from post-arrest hypoxic/anoxic coma. ${ }^{9}$

\section{Clinical Materials and Methods}

One hundred and three patients with severe head injury (GCS $\leq 8$ ) were monitored with SSEPs and continuous measurement of the EEG power spectrum as part of a standard intensive care monitoring protocol. Patients varied in age from 15 to 77 years with the mean age being 38 years. All patients were intubated and mechanically ventilated. All patients were medicated with narcotics in sedative doses and received neuromuscular blockers for intracranial pressure and ventilator management. All patients underwent ICP monitoring, almost always with an intraventricular catheter fluid-coupled to an external transducer. In a few cases a subarachnoid bolt was used to monitor ICP. In addition all patients underwent twice daily measurements of arterialjugular oxygen difference. In the latter half of the series patients also underwent twice daily measurement of cerebral blood flow using the nitrous oxide washout technique of Kety and Schmidt. ${ }^{10}$

SSEPs were recorded from the C3' and C4' electrode positions referenced to linked ears with the stimulus delivered at the contralateral median nerve. The stimuli were delivered at 2.1 Hz. with 250 stimuli averaged per tracing. Duration of the SSEP trace was $250 \mathrm{msec}$. post stimulus. Two repetitions were carried out per side and the recordings were done at approximately one hour intervals. For statistical analysis the peak to peak amplitude values for all the peaks in the SSEPs were summed from both hemispheres using a computer programme to identify peaks and sum the amplitudes. This technique has been validated previously. ${ }^{11}$ In between recordings of evoked potentials the EEG power spectrum was recorded from the F3, F4, C3, C4, P3, $\mathrm{P} 4, \mathrm{O} 1$, and $\mathrm{O} 2$ electrode positions referenced to linked ears. A 2 second time epoch was selected for measuring the power spectrum and then 50 epochs were averaged together to yield the values for each electrode position. For the purposes of this analysis the values of all electrode positions were averaged to yield a single average power spectrum at the measured time points. The average duration of monitoring was 4-5 days. Patients dropped out when they died, regained consciousness, or their monitored physiologic parameters (principally ICP) were stable over a number of days.

Patient outcomes were measured at 3,6, 12, and 24 months intervals, with the last available outcome measure used in the analysis. Outcomes were categorized according to the Glasgow Outcome Scale collapsed to 3 categories, dead/vegetative survival, severe disability, moderate disability/good outcome.

Statistical comparisons were carried out for continuous data using ANOVA for overall statistical significance. The Bonferroni method was used for individual comparisons.

\section{Results}

Figures $1 \mathrm{a}, \mathrm{b}$, and $\mathrm{c}$ show 12 hourly data points graphed for the first $108 \mathrm{hr}$. post-injury for total SSEP amplitude, percent delta (1-3 Hz.) activity in the EEG power spectrum, and total spectral amplitude. The SSEP amplitude values reveal distinct separation of the outcome groups at 7 of 9 twelve hour time epochs, unlike the values for percent delta and total EEG power (amplitude squared). For the SSEP amplitude data the differences between outcome groups are significant $(\mathrm{p}<.05)$ at 12 , $48,60,72,84,96$, and $108 \mathrm{hr}$. post injury. At all of those times 

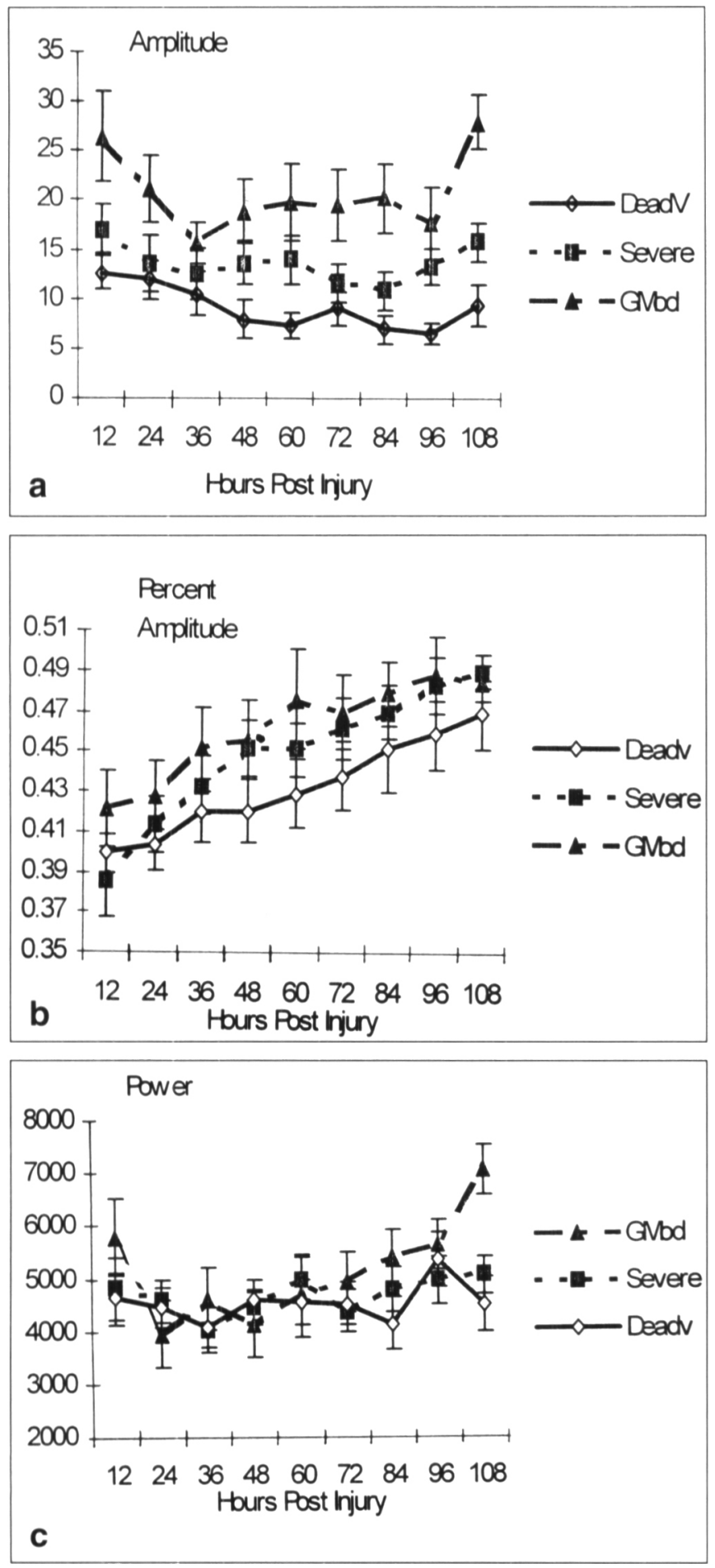

Figure 1: $a, b, c$. Figure la shows the total peak to peak SSEP amplitude measured from both hemispheres over 108 hours post injury. Values are given for mean \pm std. error. There are significant differences $(p<.05)$ between the total amplitude values for the different outcome groups at 12,48, and all time points thereafter. The difference is primarily between the good outcome/moderate disability groups and the dead vegetative groups. Figures $1 b$ and $1 c$ show the percent delta and total EEG amplitude respectively. There is no significant difference between outcome groups at any time post injury for either parameter with the single exception of total EEG amplitude at $108 \mathrm{hr}$. post injury $(p<.05)$. Gmod $=$ good outcome/moderate disability, severe $=$ severe disability, deadv $=$ dead/vegetative.
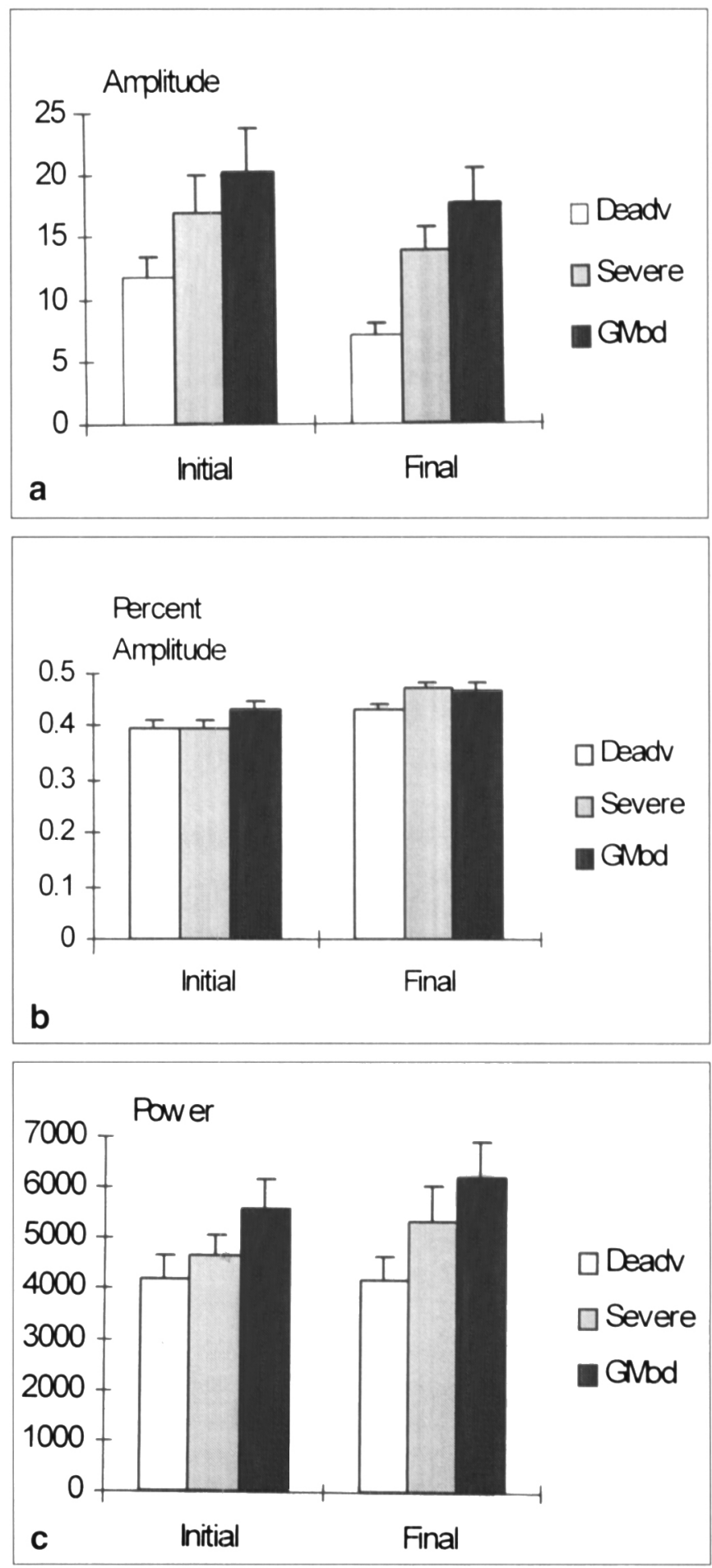

Figure 2: $a, b$, and $c$. These figures show the mean and std. errors for total SSEP amplitude, percent delta activity in the EEG, and total EEG power respectively at the initiation and termination of monitoring for patients in the 3 outcome groups. At the termination of monitoring there were significant differences between outcome groups in all 3 monitored parameters. $P=.0002, .03$, and .03 respectively for total SSEP amplitude, percent delta, and total EEG power. At the initiation of monitoring there were no significant differences. 
there is a significant difference between the good/moderate group and the dead/vegetative group. In addition, at 84 and 108 hours there is a significant difference in the total SSEP amplitude values between the good/moderate and severe groups. It is noteworthy that patients in all outcome groups had some reduction in SSEP activity over the first 48 hours post-injury. At no time post injury were there any significant differences among the outcome groups in the percent delta or total EEG power values. The total number of patients used at each time point differed because of patients dropping out due to death, recovery, or maintenance of stable intracranial physiologic parameters over a number of days.

A second analysis was carried out comparing the electrophysiologic parameters among different clinical outcome groups at the outset and termination of monitoring, in order to control for patients entering and dropping out of the monitoring process at different times after injury. The results are shown in Figures 2 $\mathrm{a}, \mathrm{b}$, and $\mathrm{c}$. None of the monitored parameters were significantly different among the various outcome groups at the initiation of monitoring, although the SSEP values came close $(p=.07)$. At the end of monitoring all three electrophysiologic parameters differed between outcome groups, $p=.0002, .03$, and .03 for total SSEP amplitude, percent delta, and total EEG power respectively. For total SSEP amplitude, there were significant differences between both the good/moderate and severe groups compared with the dead/vegetative groups. All groups had some decrease in SSEP activity over the monitoring period with the greatest decline occurring in the dead/vegetative group and the least occurring in the good/moderate group. For the total EEG power analysis, the significant difference was between the good/moderate and dead/vegetative groups. For percent delta the significant difference was between the severe group and the dead-vegetative group. At the end of monitoring the severe group had the greatest amount of slowing, the dead/vegetative group the least, and the good moderate group was intermediate.

The third analysis was done using the patients' GCS at 14 days post injury as an early index of recovery. Spearman rank correlation was used to compare initial and final electrophysiologic monitoring values against the fourteen day GCS (Table). For 37 patients who did not survive, the 14 day GCS was set arbitrarily at 3 . The lowest GCS in a patient alive at 14 days was 4. For SSEPs the final, but not initial monitoring day values correlated significantly with the 14 day GCS. Neither the initial nor final percent delta values correlated with 14 day GCS. The initial and final total EEG power correlated with the day 14 GCS.

Table: GCS - Electrophysiological correlation

\begin{tabular}{|c|c|c|c|c|}
\hline & & $\begin{array}{c}\text { SSEP } \\
\text { Amplitude }\end{array}$ & $\begin{array}{c}\text { Percent } \\
\text { Delta }\end{array}$ & $\begin{array}{l}\text { Total EEG } \\
\text { Amplitude }\end{array}$ \\
\hline \multirow{2}{*}{$\begin{array}{r}\text { Initial Spearman } r \\
p\end{array}$} & & .17 & .10 & .29 \\
\hline & $\mathrm{p}$ & .10 & .31 & .004 \\
\hline \multirow[t]{2}{*}{ Final Spearman } & $r$ & .49 & .15 & .22 \\
\hline & $\mathrm{p}$ & .0001 & .16 & .03 \\
\hline
\end{tabular}

\section{DISCUSSION}

In this series of patients we could not establish any consistent relationship between either short term outcome (14 day GCS) or longer term outcome and the relative amount of low frequency activity in the EEG. For the percent delta measurement the results were at variance with the literature in that at all times the greatest portion of slow activity was seen in the patients with good/moderate outcomes, the severe group was intermediate, and the least amount of slow activity was seen in the dead/vegetative group. For the initial - final analysis the severe group had the greatest portion of slow activity, the dead/vegetative group the least, and the good/moderate group was intermediate. This tends to reinforce the inconsistency of the relative amplitude analysis. The total EEG power results were distributed in a more intuitive fashion, with the good/moderate group having the greatest EEG power, the severe group an intermediate value, and the dead/vegetative group the least EEG power. Investigators have reported on the favorable prognostic significance of variability of the power spectrum patterns over time. ${ }^{3.4}$ However, in the acute monitoring situation, in which critical management decisions must be made within a very short time window, one may not have the luxury of several days of preceding data by which one can retrospectively gauge the degree of temporal variability. This is particularly true in the situation of acute severe head injury in which $2 / 3$ of patients who die from severe head injuries at our institution do so within $72 \mathrm{hr}$. of injury. As the results of this paper show, at any given moment in time early after injury the amount of slow activity in the EEG record cannot be used to distinguish good vs. poor prognosis patients. Nor have we found the total EEG amplitude to be a reliable indicator of an individual patient's prognosis until there is a dramatic fall in the total power at the time of electrocerebral silence. At that stage, the prognosis is usually clinically obvious by virtue of the patient's bilateral absence of pupillary reaction and often complete absence of cerebral perfusion pressure (mean arterial blood pressure - intracranial pressure). The problem with the EEG for monitoring severe head injury may lie in the fact that it is primarily a measure of spontaneous cortical neuronal activity, whereas the pathology in diffuse head injury is primarily in the white matter of the cerebral hemispheres. Measurement of coherence and phase properties of the EEG may address this deficiency. ${ }^{5}$

The prognostic capability of evoked potentials is roughly equivalent to the commonly measured clinical features (age, GCS, pupillary reactivity),, .12 so that evoked potential monitoring does not confer any special prognostic advantage in a patient who is accessible to clinical examination. It does however provide important information about patient status in patients who are pharmacologically paralyzed and sedated to facilitate respiratory or ICP management. In this situation we have frequently relied on SSEP measurement to guide therapy. For instance in patients who have lost cortical SSEP activity we will not employ heroic measures to control refractory ICP, i.e., barbiturate coma or decompressive craniectomy. In two patients who maintained intermediate and long latency SSEP activity in the face of medically uncontrollable ICP, decompressive craniectomies were carried out. In a third patient barbiturate coma was employed to control refractory ICP. One patient with a craniectomy resumed normal scholastic activities at six months post-injury. The second, older patient, returned to full independence at home, but was unable to resume work. The patient whose ICP was controlled with barbiturate coma returned to work by 2 years post injury.

The labour intensity and requirement for bedside expertise necessary to continuously monitor SSEPs discourages 
widespread use of the technique. However, one-time measurement of SSEPs may be practical for many units caring for patients with severe head injury in circumstances when patients may not be accessible to clinical examination and early information about prognosis is required.

As indicated in our results, SSEPs frequently change over time. In one series about half of patients who died from head injury and were not moribund at the outset of monitoring showed progressive deterioration of SSEP waveforms. ${ }^{7}$ Newlon et al. had previously hypothesized that such deterioration was the result of secondary insults such as raised ICP or ischemic/hypoxic insults. ${ }^{6}$ In our experience progressive deterioration of evoked potentials was usually not associated with obvious secondary insult while the patients were being monitored. In one case we did find an increase in the size of a temporal lobe contusion associated with unilateral loss of SSEP activity. Temporal lobectomy restored the SSEP activity to baseline values. Deterioration was usually unassociated with raised ICP, and when patients did succumb to raised ICP this most often followed the disappearance of SSEPs. ${ }^{13}$ The most likely explanation for this temporal sequence of events is that uncontrollable ICP may simply be a reflection of large volumes of non-viable brain in fatally injured patients. Other investigators have also observed a lack of relationship between raised ICP and evoked potential deterioration. ${ }^{14}$

Hutchinson et al. have reported the prognostic superiority of SSEPs over EEG. ${ }^{15}$ In comparing the value of on-line monitoring of SSEPs and EEG spectral analysis it is difficult to describe the relative utility of these techniques quantitatively. There is no "gold standard" against which these techniques can be validated, so that eventual patient outcome must be used as a surrogate for the absence of a contemporaneous measure. The quantitative data presented in the study do show a clear-cut separation of different outcome groups using SSEP responses at 7 of 9 twelve hour epochs post-injury whereas the EEG parameters do not show this clear cut distinction. While outcome prediction is not the point of this paper, the ability of the SSEP to distinguish between eventual outcome groups early on, and the inability of the EEG power spectrum to do so, most likely indicates the ability of the SSEP to reflect some element(s) of brain function that is/are critical to survival and outcome after severe head injury. This ability is presumably what makes the SSEP a superior monitoring tool for severe head injury. Based on the findings detailed above, we now routinely make critical management decisions based in large part on SSEP recordings. We have never done so using parameters derived from the EEG power spectrum.

\section{ACKNOWLEDGMENTS}

This work was supported in part by the Surgical Scientist Programme of the Department of Surgery. University of Toronto and by a grant from the St. Michael's Hospital Research Society.

\section{REFERENCES}

1. Stockard JJ, Bickford RG, Aung MH. The electroencephalogram in traumatic brain injury. In: Vinken PJ, Bruyn GW, eds. Handbook of Clinical Neurology, Volume 23, Injuries of the Brain and Skull Part I. Amsterdam: Elsevier, 1975; 317-367.

2. Bickford RG. Computer analysis of background activity. In: Remond E, ed. A Didactic Review of Methods and Applications of EEG. Amsterdam: Elsevier, 1977; 215-232.

3. Bricolo A, Turazzi S, Faccioli F, et al. Clinical application of compressed spectral array in long-term EEG monitoring of comatose patients. Electroencephalogr Clin Neurophysiol 1978; 45: 211 225.

4. Karnaze DS, Marshall LF, Bickford RG. EEG monitoring of clinical coma: the compressed spectral array. Neurology 1982; 32 : 289-292.

5. Thatcher RW, Cantor DS, McAlaster R, Geisler F. Krause P. Comprehensive predictions of outcome in closed head-injured patients. The development of prognostic equations. Ann $N Y$ Acad Sci 1991; 620: 82-101.

6. Newlon PG, Greenberg RP, Hyatt MS, et al. The dynamics of neuronal dysfunction and recovery following severe head injury assess with serial multimodality evoked potentials. J Neurosurg 1982; 57: 168-177.

7. Moulton R, Kresta P, Ramirez M, Tucker W. Continuous automated monitoring of somatosensory evoked potentials in posttraumatic coma. J Trauma 1991; 31: 676-685.

8. Lindsay KW, Carlin J, Kennedy I, et al. Evoked potentials in severe head injury - analysis and relation to outcome. I Neurol Neurosurg Psychiatry 1981; 44: 796-802.

9. Madl C, Grimm G, Kramer L, et al. Early prediction of individual outcome after cardiopulmonary resuscitation. Lancet 1993; 341: 855-858.

10. Kety SS, Schmidt CF. The determination of cerebral blood flow in man by the use of nitrous oxide in low concentrations. Am J Physiol 1945: 143: 53-66.

11. Moulton RJ, Konasiewicz SJ, O'Connor P. A new quantitative measure for monitoring somatosensory evoked potentials. Can J Neurol Sci 1994; 21 : S17-S22.

12. Lindsay K, Pasaoglu A, Hirst D, et al. Somatosensory and auditory brain stem conduction after head injury: a comparison with clinical features in prediction of outcome. Neurosurgery 1990;26: 278-285

13. Konasiewicz SJ, Moulton RJ, Shedden PM. Somatosensory evoked potentials and intracranial pressure in severe head injury. Can J Neurol Sci 1994; $21: 219-226$.

14. Garcia-Larrea L, Artru F, Bertrand O, et al. The combined monitoring of brain stem auditory evoked potentials and intracranial pressure in coma. A study of 57 patients. J Neurol Neurosurg Psychiatry 1992; 55: 792-798.

15. Hutchinson DO, Frith RW, Shaw NA, et al. A comparison between electroencephalography and somatosensory evoked potentials for outcome prediction following severe head injury. Electroencephalogr Clin Neurophysiol 1991; 78: 228-233. 